\title{
New Thermocouple-Based Microwave/mmWave Power Sensor MMIC Techniques in GaAs
}

\author{
Jonathan B Scott, Senior Member, IEEE, T. S. Low, Steve Cochran, Ben Keppeler, John Staroba, and Bob Yeats
}

\begin{abstract}
We describe a new RF and microwave power sensor MMIC design. The circuit incorporates a number of advances over existing designs. These include a III-V epitaxial structure optimized for sensitivity, the figure-of-merit applicable to the optimization, a mechanism for inbuilt detection of load ageing and damage to extend calibration intervals, and a novel symmetrical structure to linearize the high-power end of the scale.
\end{abstract}

Index Terms-Thermocouple, microwave, millimetre-wave, power sensor.

\section{INTRODUCTION}

$\mathbf{O}$ VER 35 years ago the first commercial RF power sensor that employed a semiconductor thermocouple was introduced [1]. Thermocouple-based sensors became the technology of choice for precision power measurement owing to their improved sensitivity, good match and excellent linearity in comparison with thermistor-based sensors [2]. The use of micromachining and the selection of materials with a large Seebeck coefficient produced a sensor with unprecedented sensitivity. Subsequently other designs for RF power sensors appeared that also relied on thermocouples [3]-[6].

In [3], the power absorber was electrically isolated from the thermocouples, and sensitivity was controlled through a mechanically different arrangement. In the original design of [1], the same materials that comprise the hot junction also form the terminating resistance. The "direct heating" method of [1] has the advantage of perfect thermal coupling between dissipator and sensor, but the desired load resistance defines the material conductivity, and this in turn sets strict limits on the doping of the semiconductor that forms one of the couple materials. In contrast, the isolated design in [3] took the alternative tradeoff of reduced sensitivity through indirect heating of the hot junctions in exchange for the possibility of higher sensitivity through freedom to adjust doping and thus the Seebeck coefficient of the couple.

Another design using indirect heating is reported in [4], [5]. Advances in this design are substantially associated with geometrical layout for improved high-frequency response and optimum sensitivity. The design employs a SiGe crystal and platinum electrodes on different substrates, chosen for their manufacturability and thermal conductivity. Reference [4] mentions the possibility of dispensing with the difficult

Jonathan Scott was with Agilent Technologies, and is now with the Department of Engineering at the University of Waikato, Hamilton, New Zealand. E-mail: jonathanscott@ieee.org.

T. Low, S. Cochran, B. Keppler, and J. Storoba are with Agilent Technologies, Santa Rosa, CA, USA.

$\mathrm{R}$ Yeats was with Agilent Technologies, and is now retired.

Manuscript received in 2010 . blocking capacitor required for signal separation in the direct heating method, but users typically prefer ac coupling because it removes the risk of damage through exposure to dc current.

Use of the indirect heating method opens up a number of possibilities. The sensor monolithic microwave integrated circuit (MMIC) described in this manuscript employs the indirect method. This design introduces three innovations available to an indirect design that are not possible with a direct one. Firstly, epitaxial material is selected in the light of the detailed physical analysis of Section III to optimize the signal-to-noise ratio (SNR) and thus dynamic range. Secondly, the circuit is electronically and geometrically symmetrical, as described in Section IV. It can work in the conventional way where thermal output voltage indicates temperature rise and thus dissipated power, but also in a "balanced mode" where a second resistor heats the "cold" junctions to set thermopile output to zero. When so balanced, the RF and secondary (dc) powers will be equal, and the power measurement is no longer subject to nonlinear thermal conductivity of substrates or thermocouple voltage, leading to a substantial improvement in linearity and reduction in calibration effort (and thus cost). Thirdly, an auxiliary circuit described in Section V is introduced to allow measurement of the terminating load resistance without otherwise disturbing the operation of the sensor. This innovation permits tracking of load resistor ageing or detection of overload-induced damage, opening the way to calibrationon-demand instead of calibration at regular intervals or when a malfunction is suspected from measured values.

\section{Thermopile Figure OF Merit}

Reference [4] states that "the indirect heating type thermocouple has the advantage that high detectivity is obtained" because "the number of thermocouples can be arbitrarily increased independent of the [load] resistor". This is a misleading view. The smallest temperature difference that can be observed above the noise (in a given bandwidth) is set not only by the output voltage of the pile for a given temperature difference, but also by the system noise. It is true that one may almost arbitrarily increase the number of thermocouples, and thus the open-circuit dc voltage to be expected for a given temperature rise. However, increasing the number of couples for fixed chip area requires that each couple be made narrower, increasing the series resistance of each couple. It turns out that this does not improve the Signal-to-Noise Ratio (SNR), and thus does not improve the minimum power dissipation that can be detected.

Assuming that the thermal noise of the thermocouple series resistance is the dominant noise contribution to the system, 
as is the case with modern amplifying electronics, it is straightforward to show that sensitivity is limited by the epitaxial layer area (so-called "chip real estate") dedicated to the thermocouples. Any advantage to increasing the number of thermocouples in the direct heating method lies in increasing the sensor output voltage in comparison with accidental thermocouples created in connecting to the external electronics. The end result of the switch to "indirect heating", all other factors ignored, has been remarkably similar dynamic range in any given sensor, as evidenced by the performance of products in the marketplace, and our thermal simulations.

In order to design an optimum material with which to fabricate thermocouples, a suitable figure of merit is required. Assume that a certain area is available between hot and cold regions in a sensor. This area may be occupied by one or more pairs of material, each pair forming a thermocouple, and all may be wired in series to form a thermopile. The width of each thermocouple bar will be inversely proportional to the number of such bars, and for a given material the resistance of each bar will be inversely proportional to its width. With no current flowing, signal voltage will be proportional to the number of bars, and total resistance will be proportional to the square of the number of bars. The noise voltage contribution from a resistance is $V_{n}=\sqrt{4 k T B R}$, where $T$ is absolute temperature, $B$ is bandwidth, $R$ is the value of the resistance and $k$ is Boltzmann's constant. It is easy to show that SNR is independent of the number of bars for a given material. Also the SNR will be maximized if the Seebeck coefficient (the voltage developed) is maximized compared to the square root of the bar material resistivity. This observation leads to a figure of merit for the material of

$$
\text { FoM }=\frac{\alpha}{\sqrt{\rho}}
$$

where $\alpha$ is the Seebeck coefficient and $\rho$ is the material bulk resistivity. ${ }^{1}$ The epitaxial materials with the largest FoM will provide the highest sensitivity, all other factors being equal.

\section{EPITAXY FOR THERMOCOUPLES}

Materials in the III-V family can show very high Seebeck coefficients. [7] In order to select the best alternative the FoM of equation (1) will be used. To this end one must calculate the open-circuit thermoelectric voltage, or Seebeck voltage, between two metal contacts placed at either end of a bar of n-type semiconductor subjected to a temperature gradient. The treatment below follows that of [8], but is expanded to include degenerate statistics and explicitly includes the temperaturedependent mobility of the semiconductor.

There are two contributions that add to produce the opencircuit voltage. One arises from the electric field within the semiconductor bar as a result of the balance of electron drift and diffusion in the presence of the temperature gradient. The

\footnotetext{
${ }^{1}$ Either bulk or sheet resistivity may be used in this case. The thickness of the epitaxial layer connects these. The thickness of the epi is limited by processing convenience and cost to about 2 microns. Since we seek to minimize the final resistance of a thermocouple bar, the maximum convenient thickness will always be used, and it is possible to consider this factor as being fixed.
}

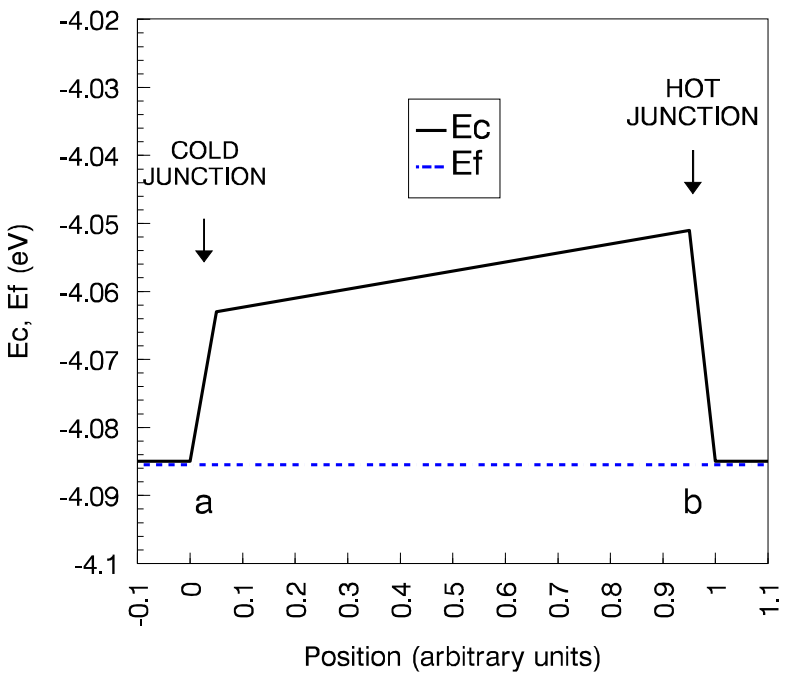

Fig. 1. Energy level diagram for the case of a bar of semiconductor material subject to a temperature gradient and with metal contacts at "cold" (a) and "hot" (b) junctions. $E_{c}$ is the conduction band edge energy, and $E_{f}$ is the Fermi energy in the semiconductor bar.

other arises from the difference in barrier heights of the two metal contacts caused by the difference in their temperatures. The energy diagram is depicted in Fig. 1. We seek first the coefficient $\alpha B=E / \frac{\partial T}{\partial z}$, the magnitude of the electric field induced by the temperature gradient divided by that gradient. We can write the current density $J$ in the bar as

$$
J=\sigma E+q \frac{\partial}{\partial z}(D n)
$$

where $\sigma$ is the material conductivity, $E$ the electric field, $q$ the charge on the electron, $z$ the distance along the bar, $D$ the electron diffusion coefficient, and $n$ the charge carrier concentration. In the case of an open circuit $J=0$, thus (2) may be expanded to:

$$
E=-\frac{q}{\sigma} \frac{\partial}{\partial z}(D n)=-\left(\frac{1}{\mu} \frac{\partial D}{\partial T}+\frac{D}{n \mu} \frac{\partial n}{\partial T}\right) \frac{\partial T}{\partial z}
$$

and so

$$
\alpha B=E / \frac{\partial T}{\partial z}=-\left(\frac{1}{\mu} \frac{\partial D}{\partial T}+\frac{D}{n \mu} \frac{\partial n}{\partial T}\right)
$$

Using the Einstein relation $D / \mu=k T / q$, equation (4) may be written in terms of the temperature-dependent electron mobility $\mu(T)$ and the carrier density $n(T)$ as

$$
\alpha B=E / \frac{\partial T}{\partial z}=-\left(\frac{k T}{q}\right)\left[\frac{\partial \ln \left(\frac{\mu k T}{q}\right)}{\partial T}+\frac{\partial \ln (n)}{\partial T}\right]
$$

In (5) the low-field electron mobility $\mu$ is a function of $n$ and $T, \mu(n, T)$, and fortunately values are well established for most semiconductors from Hall-effect measurements, for example see [9], [10]. The electron density $n(T)$ is given by the Fermi integral $\mathcal{F}_{\frac{1}{2}}$ for all doping levels $n$. [11]

$$
n(T)=N_{c} \mathcal{F}_{\frac{1}{2}}(\eta) \approx N_{c} e^{\eta}
$$


where

$$
\eta=\frac{E_{f}-E_{c}}{k T}
$$

and $E_{f}$ is the Fermi energy in the semiconductor bar, $E_{c}$ is the energy of the conduction band edge, and $N_{c}$ is the conduction band density of states. Only when $n \ll N_{c}$ does the approximation hold, and (6) with (7) reduces to $N_{c} e^{\frac{E_{f}-E_{c}}{k T}}$. As the doping level approaches $N_{c}$ the errors using Boltzmann statistics become intolerable. For many doping levels of interest, such as $n>5 \times 10^{17} \mathrm{~cm}^{-3}$ in $\mathrm{n}$-GaAs, the form with the full Fermi integral must be used. Unfortunately there is no simple algebraic expression for $\mathcal{F}_{\frac{1}{2}}(\eta)$ valid for all $\eta$. Values are available in tabular form and approximate algebraic forms exist for various ranges of $\eta$. [11], [12] These sources may be used together with equations (6) and (7) to obtain $n(T)$ and through (5) the Seebeck coefficient.

To calculate the first contribution to the thermoelectric voltage, the drift/diffusion term, the Seebeck coefficient is integrated along the n-type semiconductor bar using whatever arbitrary temperature profile $T(z)$ that may exist along the bar:

$$
V_{B}=\int_{a}^{b} E d l=\int_{a}^{b} \alpha B(T(z)) \frac{\partial T}{\partial z} d z
$$

In many practical cases of interest the temperature difference between the ends of the bar, $T_{b}-T_{a}$, is small. In these cases it is possible to assume the Seebeck coefficient is constant, or $\alpha B(T(z)) \approx \alpha B$, and that the temperature gradient is constant, or $\frac{\partial T}{\partial z}=\left(T_{b}-T_{a}\right) /(b-a)$, so that (8) reduces to:

$$
V_{B}=\int_{a}^{b} E d l \approx \alpha B\left(T_{b}-T_{a}\right)
$$

To calculate the second contribution to the thermoelectric voltage, produced by the difference in barrier heights of the two metal contacts at different temperatures, we refer again to Fig. 1. Since $J=0$ the Fermi level $E_{f}$ must be constant along the entire length of the bar, since any gradient would drive a current flow. The barrier height at the cooler junction (a), $E_{\phi}(a)$, is defined as the difference between the conduction band energy $E_{c}(a)$ and the Fermi level, so $E_{\phi}(a)=E_{c}(a)-E_{f}$. Likewise $E_{\phi}(b)=E_{c}(b)-E_{f}$. For each contact equation (6) gives

$$
\frac{n(T)}{N_{c 0} T^{\frac{3}{2}}}=\mathcal{F}_{\frac{1}{2}}(\eta)=\mathcal{F}_{\frac{1}{2}}\left(\frac{-E_{\phi}}{k T}\right)
$$

where the $T^{\frac{3}{2}}$ temperature dependence of the conduction band density of states has been explicitly factored out by defining the temperature-independent prefactor $N_{c 0}$. Implicitly differentiating both sides with respect to temperature $T$ yields

$$
-\frac{3}{2} \frac{n(T)}{N_{c 0} T^{\frac{5}{2}}}=\frac{d}{d \eta}\left(\mathcal{F}_{\frac{1}{2}}(\eta)\right) \frac{d \eta}{d T}
$$

and with equation (10) some algebra leads to

$$
-\frac{3}{2 T} \mathcal{F}_{\frac{1}{2}}(\eta)=\mathcal{F}_{\frac{1}{2}}^{\prime}(\eta) \frac{d \eta}{d T}
$$

where $\mathcal{F}_{\frac{1}{2}}^{\prime}(\eta)$ denotes the differential function as usual. Differentiating (6) with respect to $T$ yields

$$
\frac{d \eta}{d T}=-\frac{E_{\phi}}{k T} \frac{1}{T}-\frac{1}{k T} \frac{d E_{\phi}}{d T}
$$

and this may be substituted back into (12), using (7), to give

$$
\frac{k}{q}\left[\frac{3}{2} \frac{\mathcal{F}_{\frac{1}{2}}(\eta)}{\mathcal{F}_{\frac{1}{2}}^{\prime}(\eta)}-\eta\right]=\frac{1}{q} \frac{d E_{\phi}}{d T}
$$

When $n \ll N_{c}$ both $\mathcal{F}_{\frac{1}{2}}(\eta)$ and $\mathcal{F}_{\frac{1}{2}}^{\prime}(\eta)$ approach $e^{\eta}$, which would simplify this equation considerably, but as above this is not a safe approximation for many concentrations of interest.

It is now possible to assemble results and write an equation for the open-circuit voltage of the bar:

$$
V_{o / c}=\frac{E_{\phi}(a)}{q}+\int_{a}^{b} E d l-\frac{E_{\phi}(b)}{q}
$$

where the first and last terms can be obtained in the general case by numerically inverting the Fermi integral in equation (10) to get the unknown $E_{\phi}(a, b)$ from the known $n$, $T$, and $N_{c o}$ :

$$
E_{\phi}(a, b)=-k T \times \mathcal{F}_{\frac{1}{2}}^{-1}\left(\frac{n(T)}{N_{c 0} T^{\frac{3}{2}}}\right)
$$

where $\mathcal{F}_{\frac{1}{2}}^{-1}$ indicates the inverse of the Fermi integral function that must be obtained numerically. The middle term of (15) is simply $V_{B}$ obtained already in (8).

In many practical cases the temperature difference will be small and the gradient constant, in which case we can simplify $V_{o / c}$ and write it in differential form. Assume $T_{a}$ is fixed and $T_{b}=T_{a}+\Delta T$. The change in open-circuit voltage, $\Delta V_{o / c}$, can be expressed in terms of the incremental difference in temperature using the expressions derived above. We can identify the incremental increase in the bulk term, the middle term of equation (15), as

$$
\Delta V_{o / c_{2}}=\alpha B \times \Delta T
$$

where $\alpha B$ is given by (5). Similarly $\Delta V_{o / c_{1,3}}$ comes from (14):

$$
\Delta V_{o / c_{1,3}}=\Delta E_{\phi} / q=\frac{k}{q}\left[\frac{3}{2} \frac{\mathcal{F}_{\frac{1}{2}}(\eta)}{\mathcal{F}_{\frac{1}{2}}^{\prime}(\eta)}-\eta\right] \times \Delta T
$$

Now the rate of change of $V_{o / c}$ as a function of temperature difference can be written as

$$
\begin{aligned}
\frac{\Delta V_{o / c}}{\Delta T}= & \alpha B+\Delta E_{\phi} / q \\
= & -\left(\frac{k T}{q}\right)\left[\frac{\partial \ln \left(\frac{\mu k T}{q}\right)}{\partial T}+\frac{\partial \ln (n)}{\partial T}\right] \\
& +\frac{k}{q}\left[\frac{3}{2} \frac{\mathcal{F}_{\frac{1}{2}}(\eta)}{\mathcal{F}_{\frac{1}{2}}^{\prime}(\eta)}-\eta\right]
\end{aligned}
$$

This can be combined with material resistivity to obtain the material FoM.

Some open-circuit voltage measurements were taken on selected material and compared with predictions to confirm the analysis and the correct execution of the evaluation software. The proposed FoM has been evaluated for a number of candidate materials by means of the method outlined above. The predicted FoM for a selection of the materials appears in Fig. 2. It is clear that $\mathrm{n}$-InGaAs offers the best performance, at a doping concentration around $n=3 e 18 / \mathrm{cm}^{-3}$. However, 


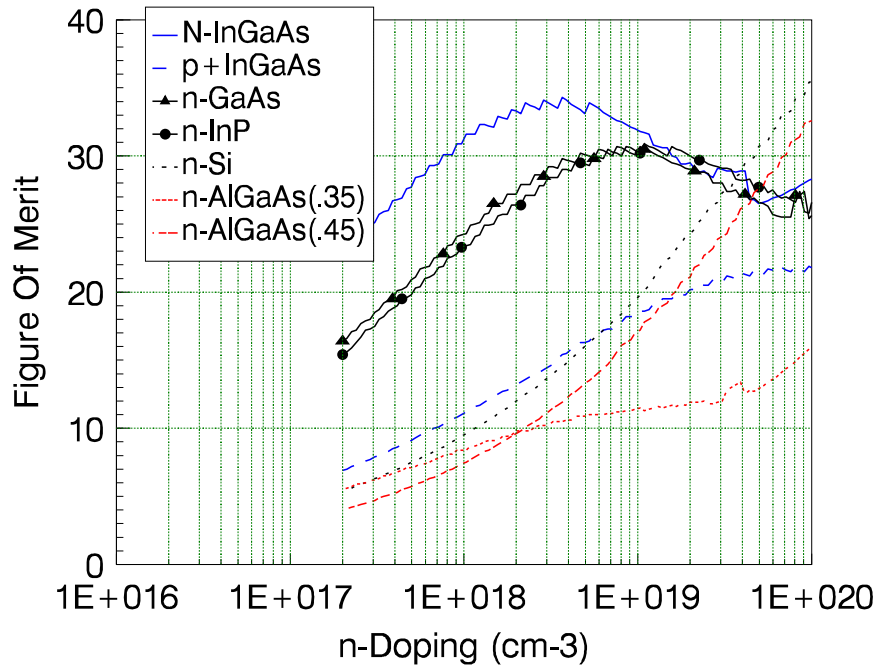

Fig. 2. Plot of Figure of Merit (FoM) against doping concentration for a variety of materials calculated as described in Section III.

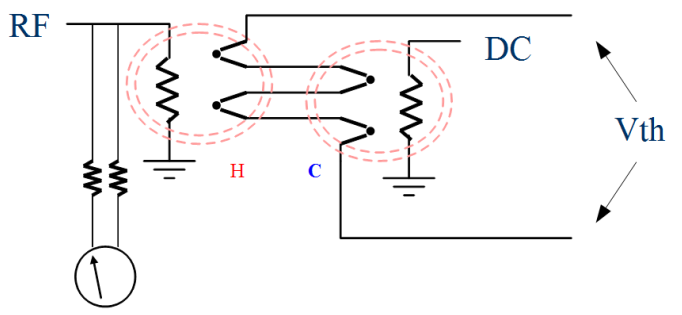

Fig. 3. Conceptual circuit schematic of the MMIC. The design consists of two power-dissipating resistors whose temperatures are compared through a series connection of several thermocouples. Additional circuitry permits monitoring of the RF load resistor.

n-GaAs offers almost as high a figure of merit. GaAs is considerably cheaper, and selective etch process technology for $\mathrm{GaAs} / \mathrm{InGaP}$ (compared with InGaAs/InP) was well established, so that was chosen for this MMIC.

\section{LAYOUT FOR BALANCED MOdE}

As intimated in the introduction, this IC embodies a novel symmetrical layout [13]. Referring to Fig. 3, there are two load resistors, one to handle RF power and a second supplied with DC power. These are of identical value equal to the characteristic impedance, typically $50 \Omega$. The thermopile is layed out so as to compare the temperatures of the two resistors. Each series of junctions, nominally called the "hot" and "cold" sets, resides on an epitaxial web as in [1], signified by the dotted ovals in the figure. For lower power levels, thermocouple linearity is not an issue, the cold junctions are indeed left cold, and the open-circuit thermocouple voltage, $V_{T H}$, is straightforwardly related to the incident power. At higher power levels, thermocouples exhibit nonlinear behaviour. This is typically partially corrected by analog or digital techniques, but ultimate accuracy suffers and calibration effort is increased.

In the design presented here, the mode of operation switches from the simple hot/cold mode to balanced mode above

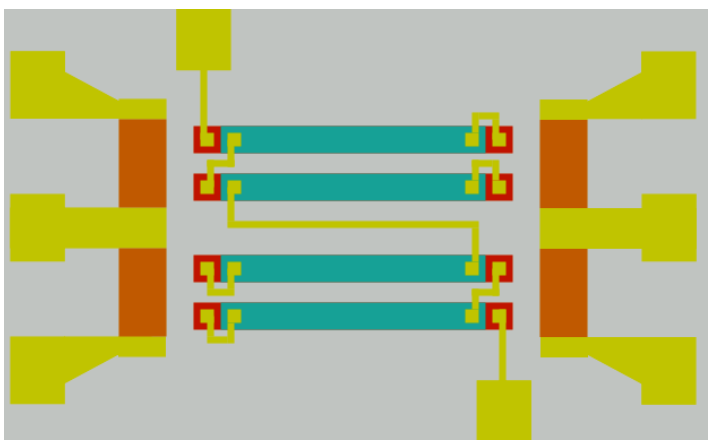

Fig. 4. Simplified layout diagram depicting the essential aspects of the MMIC required for operation in balance mode, where DC and RF powers are compared. Bond pads and coplanar manifolds may be seen at left and right ends of the circuit leading to split termination resistors. The horizontal bars in the middle comprise the thermopile. The layout may be compared to the photograph in Fig. 5.

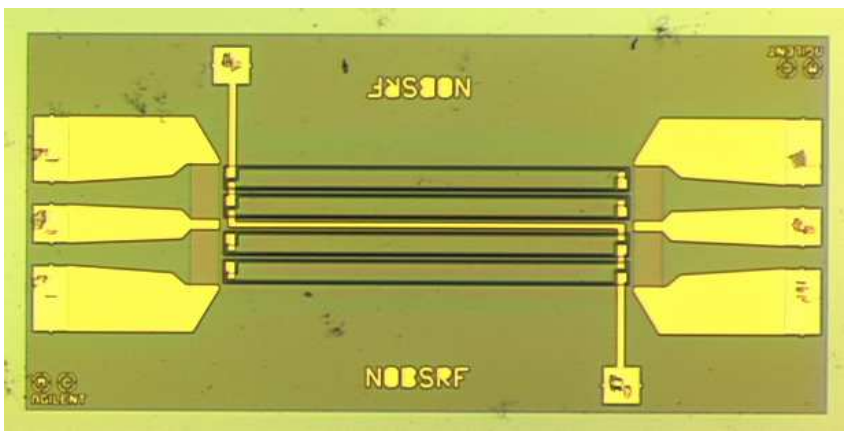

Fig. 5. Photograph of a prototype IC with symmetrical layout of thermocouples and twin loads with coplanar microwave manifolds.

a certain incident power level, before linearity affects the measurement. In the balanced mode, DC power is supplied to the second load resistor, so as to servo the thermocouple output voltage to zero. In the case of a perfectly symmetrical circuit, zero thermocouple voltage implies that the temperature of the $\mathrm{RF}$ resistor exactly equals that of the DC resistor. ${ }^{2}$ Incident RF power is then straightforwardly related to the DC power that is readily accurately measured. This "balanced" mode circumvents issues with both the linearity of thermocouple voltage and linearity of material thermal conductivity. A simplified diagram explaining the layout is given in Fig. 4. For reasons of thermal symmetry and ease of handling both resistors are layed out as two parallel resistances each of twice the desired resistance within coplanar microwave manifolds, although one of these is never used with RF energy. A photomicrograph of a prototype MMIC appears in Fig. 5.

The thermocouples depicted in Figs. 4 and 5 are a stacked design modelled on the sensors in [7]. One layer consisted of $\mathrm{n}$-doped and the second p-doped material. This design has not been used in our final design because it suffered two problems. The insulating layer between the stacked materials forms a PIN-diode style junction, and at higher temperatures and voltages it can show leakage. Also, reference to Fig. 2 shows

\footnotetext{
${ }^{2}$ If the circuit is not perfectly symmetrical, it turns out that there is a fixed ratio between the DC and RF power levels for equal temperatures and zero thermopile voltage. This is readily dealt with in calibration.
} 


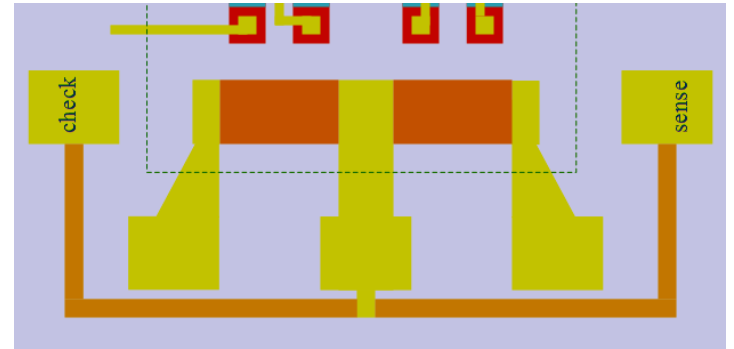

Fig. 6. Simplified layout diagram showing the idea behind the load resistor sensing arrangement. The figure shows only one of the coplanar manifolds, around which two long thin resistors have been added, leading to two additional bond pads. This schematic layout may be compared with an implementation shown in the photomicrograph in Fig. 7.

that the FoM for $\mathrm{p}$-material tends to be considerably lower than corresponding n-material, and it is possible to obtain a higher overall FoM using only one material for the semiconductor bars, and running thin metal conductors between the bars to achieve the series connections.

\section{LAYOUT FOR LOAD SENSING}

Referring back to Fig. 3, the left hand side shows a meter connected to the RF input through resistors. This is explained in more detail through the simplified layout of Fig. 6. The idea is that in normal operation the sense and check connections remain grounded. The resistors leading from the hot RF connection, the center conductor of the coplanar waveguide manifold, are relatively large and have little impact. However, a low-frequency signal can be applied to the check line, and the magnitude of that signal appearing on the load resistor can be sensed by observing the open-circuit volatge on the sense pad. The check series resistance and the load resistor form a divider, and so the load resistor value can be determined [14]. Fig. 7 presents a photomicrograph of this function implemented on a later prototype MMIC. Second-layer metal is used to access the high-value isolation resistors, but the mechanism is fundamentally the same.

Ageing and damage in thermal sensors is most commonly manifested as an increase in the value of the load resistor. Damage is usually burnout or physical breakage of the load resistor, while ageing is commonly an increase in the resistance induced by ablation of the resistor material that is accelerated at higher operating power. ${ }^{3}$ The sense scheme described here allows the meter to detect this load resistance change. Sensors were originally designed to be recalibrated anually in order to guarantee accuracy. Many users discover that their usage patterns are less demanding, and the sensors do not fall out of calibration for years. The ability of the meter to sense its own resistor opens the possibility for calibration intervals to be extended greatly, approaching a calibration-on-demand scenario. It may turn out that another mechanism, such as

\footnotetext{
${ }^{3}$ The maximum rated power of a thermal sensor is typically set by the increased wear caused by the high temperature of the load resistor. There is therefore a tradeoff between the maximum rated continuous power and the interval possible between calibrations of the sensor. In the extreme case this "wear" consists of mechanical failure through differential expansion rather than accelerated chemical change.
}

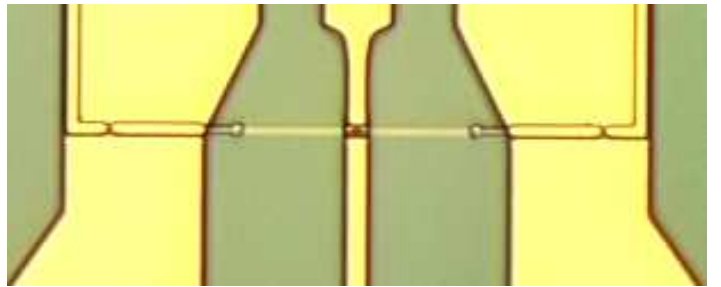

Fig. 7. Photograph of a prototype IC with load sensing resistors added to the RF manifold. The two long, high-value resistors lie between the ground and hot tracks of the coplanar waveguide manifold. The connections to the additional sensing pads are made on a separate metal layer so that the resistors may be accessed from below the coplanar ground tracks. The crossing conductors remain visible in the image, but do not connect.

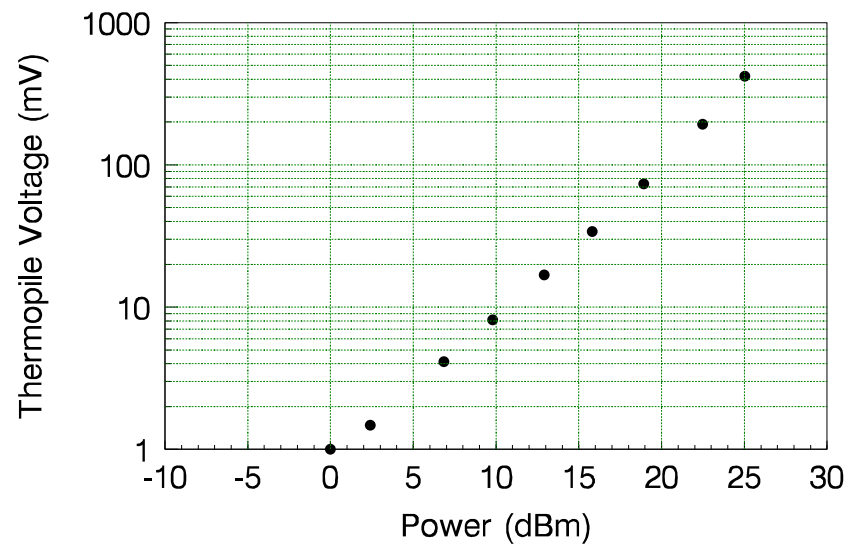

Fig. 8. Plot of thermopile voltage as a function of incident power for a backside temperature of $30 \mathrm{C}$ measured on a prototype GaAs MMIC with n-GaAs/metal thermocouples.

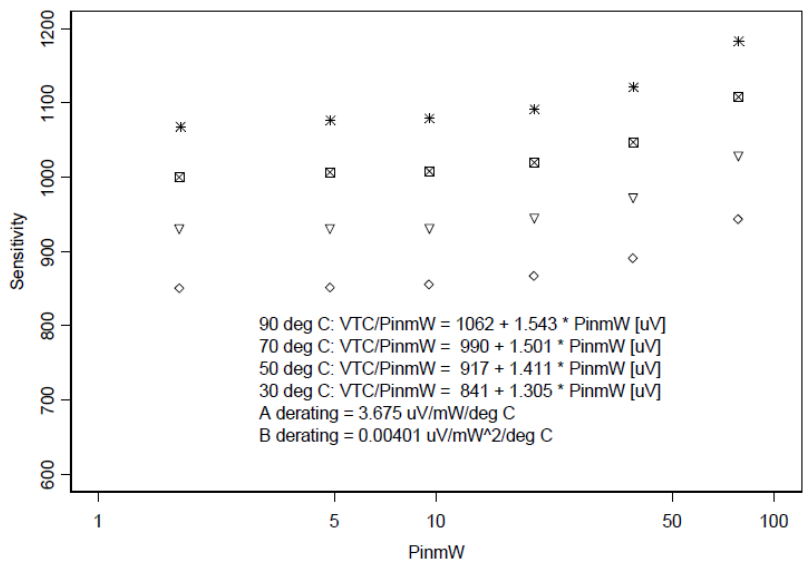

Fig. 9. Plot of the measured sensitivity in $\mu \mathrm{V} / \mathrm{mW}$ against input power in milliwatts $(\mathrm{mW})$ for various backside temperatures on the same MMIC used to obtain the data shown in Fig. 8. Data at 90C are marked with $*$, at $70 \mathrm{C}$ with $\otimes$, at $50 \mathrm{C}$ with $\nabla$ and at $30 \mathrm{C}$ with $\diamond$.

connector mechanical wear, simply takes over as the dominant cause of need for recalibration. 


\section{Vi. Measured Performance}

Fig. 8 shows the voltage measured as a function of power on a prototype circuit fabricated using $\mathrm{n}-\mathrm{GaAs}$ thermocouple bars. The sensitivity is approximately $0.85 \mathrm{~V} / \mathrm{W}$ or $850 \mu \mathrm{V} / \mathrm{mW}$ in the linear region of the response. Fig. 9 shows the sensitivity in $\mu \mathrm{V} / \mathrm{mW}$ for a variety of base temperatures. The sensitivity is considerably higher than the $160 \mu \mathrm{v} / \mathrm{mW}$ achieved in [1]. The SNR of the circuit allows reliable measurement of power down to $-33 \mathrm{dBm}$ with a settling time constant well below 1 second. The thermopile MMIC operates over a range of powers from $>100 \mathrm{~mW}$ to $<0.5 \mu \mathrm{W}$.

The dynamic range of the MMIC is set by the lowest temperature difference that can be detected above the noise, and the largest temperature that can be tolerated without mechanical damage or severely decreased reliability. This temperature range is mapped to an input power range by the thermal conductivity between the hot and cold junctions. As the epitaxial web is made bigger, the thermal resistance increases. In the limit as the substrate is etched away, the metal connections dominate the conductivity instead of the substrate. In this design the back-etch is made just large enough to reduce sensitivity of the design to etch tolerances, and transfer the responsibility for the mapping of power to temperature onto the more-tightly controlled metal patterns. This results in a minimum detectable temperature rise in the order of one thousandth of one degree, 0.001 Celsius. Mechanical damage occurs above $500 \mathrm{~mW}$ or $27 \mathrm{dBm}$, but long-term MMIC reliability constraints demand a lower continuous rated input power level of about $100 \mathrm{~mW}$ or $20 \mathrm{dBm}$. The possibility exists to use the sensor MMIC for short periods at powers higher than $20 \mathrm{dBm}$, in exchange for reduced intervals between calibration and reduced MMIC life span, especially given the capacity to track load resistor wear.

\section{CONCLUSION}

Coaxial sensors up to $67 \mathrm{GHz}$ are now using an MMIC that employs the advances presented here. The sensors employing circuits that embody the advances above offer increased sensitivity, and are expected to enable superior match, higher linearity at the top end of the dynamic range, and potentially dynamic calibration interval as features are progressively rolled out.

This manuscript

1) dispels the misconception that there is a relationship between the number of thermocouples used in an IC and the temperature measurement sensitivity that is achieved,

2) proposes a figure of merit useful for optimal selection of epitaxial material,

3) presents the physics required to accurately predict the figure of merit in compound semiconductor thermocouples,

4) identifies a suitable material that has been used to fabricate RF power sensing circuits,

5) describes a layout that enables in-situ measurement of the load resistor without compromise in RF performance, and
6) introduces a symmetrical layout and balance mode of operation that permits high natural analog linearity across the upper operating range of the IC.

The company that funded this research chooses to retain a number of design features including the RF manifold design, precise epitaxial details, and backside etch technology as trade secrets. Nevertheless, the ideas presented here are key advances in an integrated circuit that has replaced the siliconbased circuit used previously in a line of thermal power sensors.

\section{REFERENCES}

[1] Weldon H. Jackson, "A Thin-Film/Semiconductor Thermocouple for Microwave Power Measurements", HP Journal, vol. 26, no. 1, September 1974.

[2] "Fundamentals of RF and Microwave Power Measurements", Agilent Technologies Application Note 1449-2, 2003, from HP Application Note AN64-1, 1977, revised 1997.

[3] European patent 0223163B1, Hans Delfs, Rhode \& Schwarz GmbH \& Co., filed 7 November, 1985.

[4] United States Patent 6,518,743, "Wideband RF Signal Power Detecting Element and Power Detecting Device Using the Same", Setsuo Kodato, Anritsu Corporation, filed May 28, 1998.

[5] S. Kodato, T. Wakabayashi, Q. Zhuang, and S. Uchida, "New Structure for dc-60 GHz Thermal Power Sensor", paper WE3F-16, IEEE MTT$S$ International Microwave Symposium Digest, Volume 2, 17-21 June 1996, pp871-874.

[6] J. R. Kinard, D. X. Huang, and D. B. Novotny, "Performance of Multilayer Thin-Film Multijunction Thermal Converters", IEEE Transactions on Instrumentation and Measurement, vol. 44, no. 2, April 1995, pp383386.

[7] Alfons Dehe, Dimitris Pavlidis, Kyushik Hong and Hans L. Hartnagel, "InGaAs/InP Thermoelectric Infrared Sensor Using Surface Bulk Micromachining Technology", IEEE Transactions on Electron Devices, vol. 44, no. 7, July 1997, pp1052-1059.

[8] S. Hava and R. Hunsperger, "Thermo Electric properties of $\mathrm{Ga}_{x} \mathrm{Al}(1$ x)As", J. Applied Phys., vol 57, no 12, 15 June 1985.

[9] M. R. Brozel and G. E. Stillman, Properties of Gallium Arsenide, Institution of Engineering and Technology (INSPEC), October 1996.

[10] Semiconductors on NSM, Ioffe Physical Technical Institute, Russian Academy of Sciences, http://www.ioffe.ru/SVA/NSM/Semicond/, retrieved 2009.

[11] J. S. Blakemore, Semiconductor Statistics, Pergamon Press, Oxford, 1962.

[12] C. M. Wolfe, N. Holonyak, and G. E. Stillman, Physical Properties of Semiconductors, Prentice Hall Series in Solid State Physical Electronics, 1989.

[13] Jonathan B Scott, "Thermocouple microwave power sensor", United States Patent Application 20070176768, filed January 31, 2006.

[14] Jonathan B Scott, "Load measurement for a thermal microwave power sensor", United States Patent 7372249, filed January 31, 2006. 